\title{
Single-shot measurement of the spectral envelope of broad-bandwidth terahertz pulses from femtosecond electron bunches
}

\author{
J. van Tilborg, ${ }^{*}$ Cs. Tóth, N. H. Matlis, G. R. Plateau, and W. P. Leemans \\ Lawrence Berkeley National Laboratory, University of California, Berkeley, California 94720, USA \\ *Corresponding author: Jvan Tilborg@lbl.gov
}

\section{DISCLAIMER}

This document was prepared as an account of work sponsored by the United States Government. While this document is believed to contain correct information, neither the United States Government nor any agency thereof, nor the Regents of the University of California, nor any of their employees, makes any warranty, express or implied, or assumes any legal responsibility for the accuracy, completeness, or usefulness of any information, apparatus, product, or process disclosed, or represents that its use would not infringe privately owned rights. Reference herein to any specific commercial product, process, or service by its trade name, trademark, manufacturer, or otherwise, does not necessarily constitute or imply its endorsement, recommendation, or favoring by the United States Government or any agency thereof, or the Regents of the University of California. The views and opinions of authors expressed herein do not necessarily state or reflect those of the United States Government or any agency thereof or the Regents of the University of California. 


\title{
Single-shot measurement of the spectral envelope of broad-bandwidth terahertz pulses from femtosecond electron bunches
}

\author{
J. van Tilborg,* Cs. Tóth, N. H. Matlis, G. R. Plateau, and W. P. Leemans \\ Lawrence Berkeley National Laboratory, University of California, Berkeley, California 94720, USA \\ *Corresponding author: JvanTilborg@lbl.gov
}

Received February 5, 2008; revised April 16, 2008; accepted April 17, 2008; posted April 21, 2008 (Doc. ID 92410); published May 23, 2008

We present a new approach (demonstrated experimentally and through modeling) to characterize the spectral envelope of a terahertz (THz) pulse in a single shot. The coherent $\mathrm{THz}$ pulse is produced by a femtosecond electron bunch and contains information on the bunch duration. The technique, involving a single lowpower laser probe pulse, is an extension of the conventional spectral encoding method (limited in time resolution to hundreds of femtoseconds) into a regime only limited in resolution by the laser pulse length (tens of femtoseconds). While only the bunch duration is retrieved (and not the exact charge profile), such a measurement provides a useful and critical parameter for optimization of the electron accelerator. (C) 2008 Optical Society of America

OCIS codes: $020.1670,040.2235,140.7090$.

Electron accelerators such as the laser wakefield accelerator (LWFA) have recently been proven to be able to deliver electron bunches of high quality. Operation, optimization, and application of such accelerators rely among other factors, on the measurement of the bunch duration. One practical way to measure the bunch duration is through the characterization of coherent radiation [1,2] emitted by the bunches (e.g., transition radiation or diffraction radiation). The spectrum (amplitude and phase) of the coherent radiation pulse is determined by the Fourier transformation of the temporal charge profile of the electron bunch (the form factor). Because of the femtosecond nature of the bunches, the dominant part of the radiation spectrum lies in the regime of a few to tens of terahertz $(\mathrm{THz})$.

A range of characterization techniques exist to analyze the broad-bandwidth $\mathrm{THz}$ pulses. The most popular family of single-shot techniques is the one based on the electro-optic sampling (EOS) principle [3,4]. Through EOS, the $\mathrm{THz}$ field induces a change in birefringence in an electro-optic (EO) crystal. The birefringence is then probed by a near-infrared (NIR) laser pulse. Several experimental configurations exist. While in the spatial encoding geometry [5] the $\mathrm{THz}$ field is typically too weak owing to the lack of $\mathrm{THz}$ focusing, the noncollinear cross-correlation geometry $[6,7]$ is potentially complex owing to its intrinsic three-beam geometry (two laser probe beams plus the $\mathrm{THz}$ pulse) and two nonlinear processes (EO effect and frequency doubling). In another geometry (spatiotemporal mixing geometry [8]), two echelons are used to realize a temporal scan of the $\mathrm{THz}$ pulse. Note that spatial chirp could impose restrictions for shorter laser pulses $(<50 \mathrm{fs})$.

A fourth technique (spectral encoding geometry [9-11]) is based on $\mathrm{EO}$ modulation by the $\mathrm{THz}$ pulse of a chirped laser pulse. Analysis of the spectrum of the modulated laser pulse yields information of the $\mathrm{THz}$ pulse. If the spectral extent of the $\mathrm{THz}$ pulse does not exceed $1 / \sqrt{\tau_{L} \tau_{\mathrm{FL}}}$, with $\tau_{L}$ the pulse length of the chirped laser and $\tau_{\text {FL }}$ its Fourier-limited length, then the full information (amplitude and phase) of the $\mathrm{THz}$ pulse is preserved $[9,10]$. For example, with $\tau_{L}=3 \mathrm{ps}$ and $\tau_{\mathrm{FL}}=50 \mathrm{fs}$ the time resolution is limited to $\approx 400 \mathrm{fs}$. More recently it was shown [11] that the temporal resolution of this technique can be extended to $\tau_{\mathrm{FL}}$ by applying an algorithm to the spectral interference structure. However, the complex algorithm often produces unwanted numerical artifacts on the retrieved $\mathrm{THz}$ profile [8].

In this Letter we demonstrate an extention of the previously mentioned spectral encoding technique into the high-temporal-resolution domain (no algorithms). Although the phase information of the $\mathrm{THz}$ pulse is lost, its spectral envelope is retrieved in a single shot with a spectral dynamic range limited only by the inverse Fourier-limited laser pulse length $1 / \tau_{\mathrm{FL}}$. It is the spectral envelope that provides an indication of the bunch duration; therefore, it is a useful and critical parameter to measure. This new approach is less complex than the other techniques since it is based on (i) just one low-power probe laser (instead of two intense probes), (ii) a spectrometer that is fairly alignment insensitive (instead of a noncollinear cross correlator or relay-imaged echelons), and (iii) a simple Fourier transformation to retrieve the $\mathrm{THz}$ spectrum (instead of a complex algorithm). Verification of the validity of this technique based on a model and experiments is presented next.

In the LWFA electron bunches are produced through the interaction of an intense focused NIR laser with a plasma. Coherent $\mathrm{THz}$ radiation is emitted as the bunches exit the plasma-vacuum boundary. The details on the specific geometry of the LWFA used for the experiments described in this work, as well as their $\mathrm{THz}$ emission, have been presented in previous work $[1,2,7,12]$. The setup is sketched in Fig. 1. Part of the LWFA-produced $\mathrm{THz}$ emission is collected by an $F / 2$ off-axis parabola and focused out of the vacuum chamber onto an EO crystal (200- $\mu$ m-thick GaP or ZnTe). As seen in Fig. 1, a 


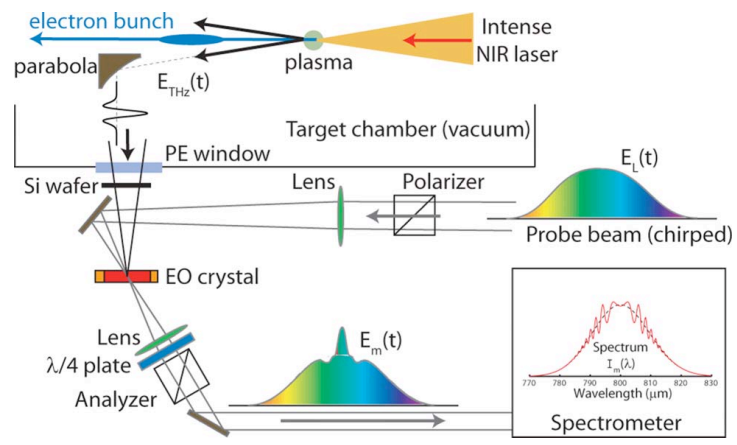

Fig. 1. (Color online) Few-cycle THz pulse $E_{\mathrm{THz}}(t)$ is emitted as LWFA-produced electron bunches exit the plasmavacuum boundary. The $\mathrm{THz}$ field induces birefringence in the EO crystal, which is probed by a chirped laser pulse $E_{L}(t)$. The modulated laser spectrum $I_{m}(\lambda)=\left|E_{m}(\lambda)\right|^{2}$ contains information of the $\mathrm{THz}$ pulse.

chirped low-power NIR probe laser $E_{L}(t)$ (linearly polarized) is directed and focused through the same EO crystal. The field profile for $E_{L}(t)$ is defined as

$$
E_{L}(t)=\exp \left[\frac{(1-\iota b / 2) t^{2}}{2 \tau_{\mathrm{FL}}^{2}\left(1+b^{2} / 4\right)}\right] \exp \left[\iota \omega_{0} t\right],
$$

with $b$ the linear chirp parameter and $\omega_{0}$ the central angular frequency. The instanteneous frequency $\omega_{\text {inst }}(t)$ of the chirped laser pulse is given by

$$
\omega_{\text {inst }}(t)=\omega_{0}+\frac{b}{2 \tau_{\mathrm{FL}}^{2}\left(1+b^{2} / 4\right)} t .
$$

The single-cycle $\mathrm{THz}$ waveform $E_{\mathrm{THz}}(t)$ incident on the EO crystal induces a time-dependent birefringence with a phase shift between the fast and slow axis given by $\Gamma_{\mathrm{THz}}(t)$. It can be shown [12] that in the frequency domain $\Gamma_{\mathrm{THz}}(\omega) \propto E_{\mathrm{THz}}(\omega) T_{\text {crystal }}(\omega)$, with $T_{\text {crystal }}(\omega)$ being the effects in the EO crystal such as group velocity mismatch, dispersion, and absorption. The $\lambda / 4$ plate (see Fig. 1) allows the user to change the polarization state of the probe beam, depending on the angle $\delta$ between the plate's axis and the laser polarizion (linear for $\delta=0$, circular for $\delta=\pi / 4$, and elliptical for $0<\delta<\pi / 4)$. As indicated in Fig. 1, the $\mathrm{THz}$-modulated laser profile transmitted through the cross-polarized analyzer is labeled $E_{m}(t)$, with its field profile given by

$$
\begin{aligned}
E_{m}(t)= & \frac{1}{\sqrt{2}} E_{L}(t)[-\cos \delta \cos \xi+\iota \sin \delta \sin \xi \\
& \left.+\cos \delta \sin \xi e^{\mathrm{I} \Gamma_{\mathrm{THz}}(t)}+\iota \sin \delta \cos \xi e^{\mathrm{I} \Gamma_{\mathrm{THz}}(t)}\right]
\end{aligned}
$$

with $\xi=\pi / 4-\delta$. Note that for circular polarized light Eq. (3) reduces to

$$
I_{m}(t)=\left|E_{m}(t)\right|^{2}=\left|E_{L}(t)\right|^{2}\left[1+\sin \Gamma_{\mathrm{THz}}(t)\right] / 2 .
$$

As is illustrated in Fig. 1, a spectrometer (600 grooves $/ \mathrm{mm}$ with a $100 \mu \mathrm{m}$ opening slit) was used to record the modulated spectrum $I_{m}(\lambda)$, with $\lambda$ being the wavelength. The inset shows that the modulated spectum $I_{m}(\lambda)$ contains multiple oscillations, as expected for $\mathrm{THz}$ pulses with a time structure shorter than $\sqrt{\tau_{L} \tau_{\mathrm{FL}}}$. The modulated spectrum $I_{m}(\lambda)$ can be normalized through $I_{m, \text { norm }}(\lambda)$ $=\left[I_{m}(\lambda)-I_{\text {ref }}(\lambda)\right] / I_{\text {ref }}(\lambda)$, with $I_{\text {ref }}(\lambda)$ being the reference spectrum in the absence of any $\mathrm{THz}$ fields.

Modeled spectral interferograms are depicted in Fig. 2(a). For these curves the profile $\Gamma_{\mathrm{THz}}(t)$ was modeled based on $E_{\mathrm{THz}}(\omega)=F(\omega)=\exp \left(-\tau_{e}^{2} \omega^{2} / 2\right)$, with $F(\omega)$ being the form factor of the electron bunch and $\tau_{e}=100 \mathrm{fs}$ being the Gaussian rms bunch length [for simplification crystal effects are momentarily ignored $\left.\left(T_{\text {crystal }}=1\right)\right]$. The amplitude of $\Gamma_{\mathrm{THz}}(t)$ was set at $0.1 \mathrm{rad}$. The solid black curves in Fig. 2 correspond to $\left(\tau_{\mathrm{FL}}, \delta, b\right)=(40 \mathrm{fs}, \pi / 4,150)$, the dotted curves to $(50 \mathrm{fs}, \pi / 16,150)$, and the solid gray curves to (50 fs , $\pi / 16,250)$.

Through Eq. (2) one can convert the wavelength parameter $\left(\lambda=2 \pi c / \omega_{\text {inst }}\right)$ to a "new" time axis $t^{*}$. Doing so for $I_{m, \text { norm }}(\lambda)$, the new profile is labeled $I_{m \text {,inst }}\left(t^{*}\right)$. A Fourier transformation of $I_{m \text {,inst }}\left(t^{*}\right)$ yields $\left|I_{m, \text { inst }}\left(\nu^{*}\right)\right|$, with $\omega^{*}=2 \pi \nu^{*}$. The absolute spectra $\left|I_{m \text {,inst }}\left(\nu^{*}\right)\right|$ for the three curves of Fig. 2(a) are plotted in Fig. 2(b). Also plotted in Fig. 2(b) as the black dashed curve is the $\mathrm{THz}$ spectrum $\left|\Gamma_{\mathrm{THz}}(\nu)\right|$. One can see that the envelopes of the oscillatory curves $\left|I_{m \text {,inst }}\left(\nu^{*}\right)\right|$ match the $\mathrm{THz}$ spectrum $\left|\Gamma_{\mathrm{THz}}(\nu)\right|$. It is because of this matching that analysis of the spectral inteferogram results in single-shot information of the $\mathrm{THz}$ spectrum. This new result has been extensively studied and is found to be true for a wide parameter range for $b$, the amplitude of $\Gamma_{\mathrm{THz}}(t), \tau_{L}, \tau_{\mathrm{FL}}$, and $\tau_{e}$, providing that $\tau_{e}>\tau_{\mathrm{FL}}$ and that $\Gamma_{\mathrm{THz}}(t)$ does not exceed $\simeq 0.3 \mathrm{rad}$. Note that this technique cannot resolve spectral substructure, such as modulations owing to absorption lines or the presence of multiple $\mathrm{THz}$ pulses. Also, we verified that the presented technique is equally valid following the EO modulation description of Jamison et al. [4], where $E_{m}(t)$ $=E_{L}(t)+a(d / d t)\left[E_{L}(t) E_{\mathrm{THz}}(t)\right]$.

To experimentally verify the modeled predictions we applied the setup in Fig. 1 to the $\mathrm{THz}$ pulses from
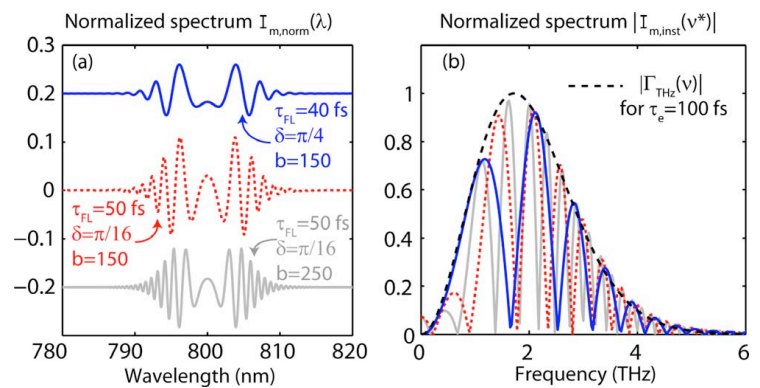

Fig. 2. (Color online) (a) Modeled spectral interferograms $I_{m, \text { norm }}(\lambda)$ for various parameters for $\tau_{\mathrm{FL}}, \delta$, and $b$. Through the wavelength-to-time parameter substitution $\lambda \rightarrow t^{*}$ in $I_{m, \text { norm }}(\lambda)$ the function $I_{m \text {,inst }}\left(t^{*}\right)$ is obtained, with its Fourier transformation $\left|I_{m \text {,inst }}\left(\nu^{*}\right)\right|$ plotted in (b). Note that the envelopes of the three curves in (b) match the original $\mathrm{THz}$ spectrum $\left|\Gamma_{\mathrm{THz}}(\nu)\right|$ (black dashed curve). 
the LWFA. Each modulated laser spectrum was recorded with a $512 \times 512$ pixel 16 -bit camera with a resolution of $0.11 \mathrm{~nm} /$ pixel. Two typical measured normalized spectral interferograms $I_{\text {norm }}(\lambda)$ are plotted in Figs. 3(b) and 3(d), based on 200- $\mu$ m-thick $\mathrm{ZnTe}$ and GaP EO crystals, respectively. The parameters for the probe laser were independently measured and were found to be $\tau_{\mathrm{FL}}=40 \mathrm{fs}(\mathrm{rms})$ and $b$ $=150$. The laser was near-linearly polarized based on $\delta=26 \mathrm{mrad}$ to increase the signal-to-noise ratio [13]. After the conversion $I_{\text {norm }}(\lambda) \rightarrow I_{\text {inst }}\left(t^{*}\right)$, the Fouriertransformed curves $\left|I_{m \text {,inst }}\left(\nu^{*}\right)\right|$ are displayed in Figs. 3(a) and 3(c). Also depicted in Figs. 3(a) and 3(c) as black dashed curves is the spectrum of the $\mathrm{THz}$ pulse $\left|\Gamma_{\mathrm{THz}}(\nu)\right|=\left|E_{\mathrm{THz}}(\nu) T_{\text {crystal }}(\nu)\right|$. Here the crystal effects [12] were not ignored. Note that the spectral cutoff [as can been seen in the data in Fig. 3(a)] for the $\mathrm{ZnTe}$ crystal is $\nu=4.1 \mathrm{THz}$. The function $T_{\text {crystal }}(\nu)$ for GaP has a cutoff at a higher frequency $(\nu$ $=7-8 \mathrm{THz}$ ), making it more suitable to study $50 \mathrm{fs}$ type electron bunches [for which $F(\nu)$ extends to $\nu$ $=\omega / 2 \pi \sim 3-4 \mathrm{THz}]$. It has been shown $[12,14]$ that the LWFA-produced THz field $E_{\mathrm{THz}}(\nu)$ can be modeled as $E_{\mathrm{THz}}(\nu)=D(\nu) F(\nu)$. The diffraction effects $D(\nu)$ are now included and are based on a transverse plamsa
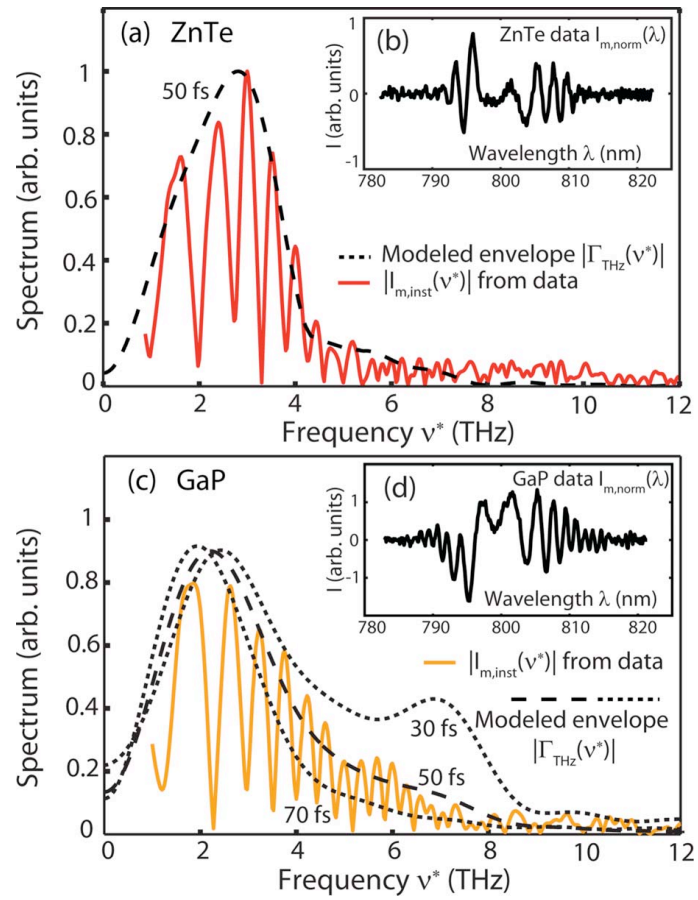

Fig. 3. (Color online) Experimentally obtained modulated laser spectra $I_{m}(\lambda)$ are normalized [yielding $I_{m, \text { norm }}(\lambda)$ ] and plotted in (b) and (d). After the coordinate substitution $\lambda$ $\rightarrow t^{*}$ [yielding $I_{m \text {,inst }}\left(t^{*}\right)$ ], the Fourier-transformed curves $\left|I_{m, \text { inst }}\left(\nu^{*}\right)\right|$ are plotted in (a) and (c). Also displayed in (a) and (c) as the black dashed curves are the $\mathrm{THz}$ spectra $\left|\Gamma_{\mathrm{THz}}(\nu)\right|$, based on coherent emission from a $50 \mathrm{fs}(\mathrm{rms})$ electron bunch. Modeled curves for a 30 and $70 \mathrm{fs}$ bunch are also shown in (c) to highlight the optimum fit for a $50 \mathrm{fs}$ bunch. size of $\simeq 150 \mu \mathrm{m}$, as has been previously discussed $[12,14]$. The black dashed curves in Figs. 3(a) and 3(c) for $\left|\Gamma_{\mathrm{THz}}(\nu)\right|$ are in good agreement with the data the envelope of $\left.\left|I_{m \text {,inst }}\left(\nu^{*}\right)\right|\right]$. The fit was optimized by only varying $\tau_{e}$ to $50 \mathrm{fs}$ (rms). This value is in excellent agreement with the bunch duration obtained on the same LWFA with alternative EOS-based techniques $[2,7,12]$.

In conclusion, we have demonstrated the extension of the EOS spectral encoding technique to a larger spectral dynamic range (only limited by $\sim 1 / \tau_{\mathrm{FL}}$ ). Although this technique does not capture phase information of $E_{\mathrm{THz}}(\nu)$, it does allow for determination of the envelope of $\left|E_{\mathrm{THz}}(\nu)\right|$, which makes it a useful diagnostic for femtosecond electron bunches. The LWFA electron bunches were demonstrated to have a duration of $50 \mathrm{fs}$ ( $\mathrm{rms}$ ), which was confirmed by alternative methods. This technique has the advantages that it requires only one low-power laser pulse, a spectrometer (easy to align), while no algorithms are needed.

This work was supported by the U.S. Department of Energy and the Defense Advanced Research Projects Agency (DARPA). We acknowledge fruitful discussions with B. Yellampalle and K. Y. Kim.

\section{References}

1. W. P. Leemans, C. G. R. Geddes, J. Faure, Cs. Tóth, J. van Tilborg, C. B. Schroeder, E. Esarey, G. Fubiani, D. Auerbach, B. Marcelis, M. A. Carnahan, R. A. Kaindl, J. Byrd, and M. C. Martin, Phys. Rev. Lett. 91, 074802 (2003).

2. J. van Tilborg, C. B. Schroeder, C. V. Filip, Cs. Tóth, C. G. R. Geddes, G. Fubiani, R. Huber, R. A. Kaindl, E. Esarey, and W. P. Leemans, Phys. Rev. Lett. 96, 014801 (2006).

3. G. Gallot and D. Grischkowsky, J. Opt. Soc. Am. B 16, 1204 (1999).

4. S. P. Jamison, A. M. MacLeod, G. Berden, D. A. Jaroszynski, and W. A. Gillespie, Opt. Lett. 31, 1753 (2006).

5. J. Shan, A. S. Weling, E. Knoesel, L. Bartels, M. Bonn, A. Nahata, G. A. Reider, and T. F. Heinz, Opt. Lett. 25, $426(2000)$.

6. S. P. Jamison, J. Shen, A. M. MacLeod, W. A. Gillespie, and D. A. Jaroszynski, Opt. Lett. 28, 1710 (2003).

7. J. van Tilborg, C. B. Schroeder, Cs. Tóth, C. G. R. Geddes, E. Esarey, and W. P. Leemans, Opt. Lett. 32, 313 (2007).

8. K. Y. Kim, B. Yellampalle, A. J. Taylor, G. Rodriguez, and J. H. Glownia, Opt. Lett. 32, 1968 (2007).

9. F. G. Sun, Z. Jiang, and X.-C. Zhang, Appl. Phys. Lett. 73, 2233 (1998).

10. Z. Jiang and X.-C. Zhang, Appl. Phys. Lett. 72, 1945 (1998).

11. B. Yellampalle, K. Y. Kim, G. Rodriguez, J. H. Glownia, and A. J. Taylor, Appl. Phys. Lett. 87, 211109 (2005).

12. J. van Tilborg, C. B. Schroeder, C. V. Filip, Cs. Tóth, C. G. R. Geddes, G. Fubiani, E. Esarey, and W. P. Leemans, Phys. Plasmas 13, 056704 (2006).

13. Z. Jiang, F. G. Sun, Q. Chen, and X.-C. Zhang, Appl. Phys. Lett. 74, 1191 (1999).

14. C. B. Schroeder, E. Esarey, J. van Tilborg, and W. P. Leemans, Phys. Rev. E 69, 016501 (2004).

\section{This work was supported by the U.S. Department of Energy under Contract No. DE-AC02-05CH11231,}

